\title{
Vortex Solitons for a Class of Schrödinger Equation with Square Root Nonlinear Term
}

\author{
Weikang Chen \\ College of Sciences, University of Shanghai for Science and Technology, Shanghai, China \\ Email: 870084315@qq.com
}

How to cite this paper: Chen, W.K. (2020) Vortex Solitons for a Class of Schrödinger Equation with Square Root Nonlinear Term. Advances in Pure Mathematics, 10, 174-180.

https://doi.org/10.4236/apm.2020.104011

Received: March 22, 2020

Accepted: April 6, 2020

Published: April 9, 2020

Copyright $\odot 2020$ by author(s) and Scientific Research Publishing Inc. This work is licensed under the Creative Commons Attribution International License (CC BY 4.0).

http://creativecommons.org/licenses/by/4.0/

\begin{abstract}
In this paper, we prove an existence theory for ring-profiled optical vortex solitons via constrained minimization, which are considered in the context of an electromagnetic light wave propagating in a nonlinear media and governed by a nonlinear Schrödinger type equation with square root nonlinear term.
\end{abstract}

\section{Keywords}

Optical Vortex Solitons, Square Root Nonliear, Constrained Minimization

\section{Introduction}

In optics research, a fundamental prototype situation is that the light waves are described by a complex-valued wave function governed by nonlinear Schrödinger equations [1]-[8]. These rigorous mathematical treatments of such nonlinear problems provide more possibilities for the existence and properties of optical vortices. Our interest is motivated by the work of Lin, Belić, Petrović, Hajaiej and Chen [9], the mathematical analysis of Lin and Ren [10].

In dimensionless form, consider the following nonlinear Schrödinger equation,

$$
i \partial_{z} E+\frac{1}{2} \nabla_{\perp}^{2} E+f(I) E=0,
$$

where $E$ is the evolution of the slowly varing electric field envelope propagating in the longitudinal $z$-direction; $\nabla_{\perp}^{2}$ is the Laplace operator over the transverse plane of coordinates $(x, y)$ which is perpendicular to the $z$-axis. The function $f$ depends on the total field intensity, I, i.e. $I=|E|^{2}$, and we will concentrate henceforth on the model of the self-focusing square-root nonlinearity.

$$
f\left(|E|^{2}\right)=1-\frac{1}{\sqrt{1+|E|^{2}}},
$$


which describes narrow-gap semiconductors [11] [12].

We focus on spatial optical solitons. Spatially localized solutions of (1), which do not change their intensity profile during propagation, can be described under the spatial soliton ansatz

$$
E(r, \theta, z)=u(r) \mathrm{e}^{i(n \theta+\alpha z)}
$$

where $r$ and $\theta$ are real polar coordinates over $\mathbb{R}^{2}$, and $r=\sqrt{x^{2}+y^{2}}$, $\theta=\arctan (y / x), u(r)$ is the radial profile function which gives rise to the intensity of light waves, $n \in \mathbb{Z}$ is the winding number, and $\alpha \in \mathbb{R}$ is the wave propagation constant. This ansatz describe a vortex wave centered around the $z$-axis. Inserting (3) into (1) and in a square root nonlinear media, we arrive at the following equation

$$
\left(r u_{r}\right)_{r}-\frac{n^{2}}{r} u+2 r u-\frac{2 r u}{\sqrt{1+u^{2}}}-2 \alpha r u=0
$$

Due to the presence of the vortex core, in other words, the regularity of $u$ at $r=0$, we impose the condition $u(0)=0$. Besides, such ring-like beams remain localized that allows us to mathematically impose the "boundary" condition $u(R)=0$ for $R>0$ sufficiently large, where $R$ represents the distance from the vortex core.

Therefore, in view of (4), we can get the $n$-vortex equation with boundary conditions.

$$
\left\{\begin{array}{l}
\left(r u_{r}\right)_{r}-\frac{n^{2}}{r} u+2 r u-\frac{2 r u}{\sqrt{1+u^{2}}}-2 \alpha r u=0 \\
u(0)=0, u(R)=0
\end{array}\right.
$$

In this paper, we treat (5) as a nonlinear eigenvalue problem and prove the existence of positive solution pairs $(u, \alpha)$ by a constrained minimization approach, with a prescribed energy flux constrained.

\section{Preliminary Setting and Main Theorems}

In this section, we give some basic notations and lemmas which will be used in next section. In order to approach the Equation (5), we write down the action functional $I_{\alpha}: H \rightarrow \mathbb{R}$ defined as

$$
I_{\alpha}(u)=\frac{1}{2} \int_{0}^{R}\left\{r u_{r}^{2}+\frac{n^{2}}{r} u^{2}-2(1-\alpha) r u^{2}+4 r \sqrt{1+u^{2}}\right\} d r
$$

where $|n| \geq 1, H$ is the completion of

$$
X=\left\{u \in C^{1}[0, R] \mid u(0)=0=u(R)\right\}
$$

equipped with the inner product

$$
(u, v)=\int_{0}^{R}\left\{r u_{r} v_{r}+\frac{1}{r} u v\right\} \mathrm{d} r, u, v \in H
$$

As a Hilbert space, $H$ may be considered as an embedded subspace of 
$W_{0}^{1,2}\left(B_{R}\right)$ which is composed of radially symmetric functions such that any element $u \in H$ enjoys the desired property $u(0)=0$, where $B_{R}:=\left\{(x, y) \in \mathbb{R}^{2}: x^{2}+y^{2} \leq R^{2}\right\}$.

Lemma 2.1. From the inequalities

$$
\left(\sqrt{1+u^{2}}-1\right)^{2} \leq \frac{1}{4} u^{4} \text { for } u \in \mathbb{R}
$$

and

$$
\int_{0}^{R} r u^{2} \mathrm{~d} r \leq R^{2} \int_{0}^{R} \frac{u^{2}}{r} \mathrm{~d} r
$$

we get that there exists a constant $C>0$, such that $I_{\alpha}(u) \leq C\|u\|_{H}^{2}$.

For convenience, we define the "energy" functional as

$$
\varepsilon(u)=\frac{1}{2} \int_{0}^{R}\left\{r u_{r}^{2}+\frac{u^{2}}{r}+4 r \sqrt{1+u^{2}}\right\} \mathrm{d} r
$$

Now, we state our main theorem in this paper.

Theorem 2.2. For any parameters $|n| \geq 1$, consider the $\mathrm{n}$-vortex Equation (5) with boundary conditions, describing ring-profile vortex solitons in a square-root nonlinear media, with the prescribed energy flux $\Phi(u)=\Phi_{0}>0$, and $R>0$.

1) There exists a solution pair $(u, \alpha)$ with $u(r)>0, r \in(0, R)$ and $\alpha \in \mathbb{R}$.

2) For $r \in[0, R]$, the energy flux $\Phi(u)=\Phi_{0} \leq \frac{1}{4}$, and there exists no nontrivial solution, if $n^{2}+2 r^{2} \alpha>0$.

\section{Existence of Vortices via Constrained Minimization}

In this section, we consider the wave propagation constant $\alpha$ as a Lagrange multiplier, we prove the existence of solution of the Equation (5) with constrained minimization approach.

We rewrite the $n$-vortex Equation (5) as

$$
\left\{\begin{array}{l}
\left(r u_{r}\right)_{r}-\frac{n^{2}}{r} u+2 r u-\frac{2 r u}{\sqrt{1+u^{2}}}=2 \alpha r u \\
u(0)=0, u(R)=0
\end{array}\right.
$$

Define the function $I$ and the soliton energy flux as

$$
\begin{gathered}
I(u)=\frac{1}{2} \int_{0}^{R}\left\{r u_{r}^{2}+\frac{n^{2}}{r} u^{2}-2 r u^{2}+4 r \sqrt{1+u^{2}}\right\} \mathrm{d} r \\
\Phi(u)=\int_{0}^{2 \pi} \mathrm{d} \theta \int_{0}^{R} r u^{2} \mathrm{~d} r=2 \pi \int_{0}^{R} r u^{2} \mathrm{~d} r
\end{gathered}
$$

Thus, to get a solution of (12), it suffices to show that a solution to the following exists:

$$
\min \left\{I(u) \mid u \in \Lambda, \Phi(u)=\Phi_{0}\right\}, \Phi_{0}>0
$$

where the nonempty admissible class $\Lambda$ is defined by 
$\Lambda=\{u(r)$ is absolutely continuous over $[0, R], u(0)=u(R)=0, \varepsilon(u)<\infty\}$

with $\varepsilon(u)$ being defined by (11).

The proof of Theorem 2.2. 1) Using the energy flux $\Phi_{0}$, we have

$$
I(u) \geq \frac{1}{2}\left(\int_{0}^{R} r u_{r}^{2} \mathrm{~d} r+n^{2} \int_{0}^{R} \frac{u^{2}}{r} \mathrm{~d} r\right)-\frac{\Phi_{0}}{2 \pi}
$$

Let $\left\{u_{m}\right\}$ be a minimizing sequence of (13). Then (15) gives the bound

$$
\int_{0}^{R} r u_{m, r}^{2} \mathrm{~d} r+\int_{0}^{R} \frac{u_{m}^{2}}{r} \mathrm{~d} r \leq C
$$

where $C>0$ is a constant independent of $m$. We know the fact that the distributional derivative of $u$ must satisfy $\left.|| u\right|_{r}|\leq| u_{r} \mid$, and the functionals $I$ and $\Phi$ are even. Thus, we may assume that the sequence $\left\{u_{m}\right\}$ consists of non-negative valued functions. Therefore, it is clear that we may view these functions as radially symmetric over the disk $B_{R}$ and vanishing on its boundary. Moreover, with (16) and (10), it can be seen that $\left\{u_{m}\right\}$ belongs in $W_{0}^{1,2}\left(B_{R}\right)$ under the reduced norm,

$$
\|u\|^{2}=\int_{0}^{R} r u^{2} \mathrm{~d} r+\int_{0}^{R} r u_{r}^{2} \mathrm{~d} r
$$

Therefore, $\left\{u_{m}\right\}$ is bounded in $W_{0}^{1,2}\left(B_{R}\right)$. Without loss of generality, we get the weak convergence of $\left\{u_{m}\right\}$ to an element $u \in W_{0}^{1,2}\left(B_{R}\right)$. Using the compact embedding $W_{0}^{1,2}\left(B_{R}\right) \rightarrow L^{p}\left(B_{R}\right)$ for $p \geq 1, \quad u_{m} \rightarrow u$ strongly in $L^{p}\left(B_{R}\right)$ as $m \rightarrow \infty$. Hence, $u$ is radially symmetric as well with $u(R)=0$.

In view of (16) and Fatou's lemma, Let $(X, \Sigma, \mu)$ be a measure space and $\left\{f_{n}: X \rightarrow[0, \infty)\right\}$ a sequence of nonnegative measurable functions. Then the function $\liminf _{n \rightarrow \infty} f_{n}$ is measurable and

$$
\int_{X} \liminf _{n \rightarrow \infty} f_{n} \mathrm{~d}_{\mu} \leq \liminf _{n \rightarrow \infty} \int_{X} f_{n} \mathrm{~d}_{\mu}
$$

we have

$$
\begin{gathered}
\int_{0}^{R} r u_{r}^{2} \mathrm{~d} r \leq \liminf _{m \rightarrow \infty} \int_{0}^{R} r u_{m, r}^{2} \mathrm{~d} r \\
\int_{0}^{R} \frac{u^{2}}{r} \mathrm{~d} r \leq \liminf _{m \rightarrow \infty} \int_{0}^{R} \frac{u_{m}^{2}}{r} \mathrm{~d} r \\
\int_{0}^{R} 2 r \sqrt{1+u^{2}} \mathrm{~d} r \leq \liminf _{m \rightarrow \infty} \int_{0}^{R} 2 r \sqrt{1+u_{m}^{2}} \mathrm{~d} r
\end{gathered}
$$

Therefore, from (10) and (19)-(21), we get

$$
I(u) \leq \liminf _{m \rightarrow \infty} I\left(u_{m}\right)
$$

Following as in [13]. Let $\left\{u_{m}\right\}$ be a sequence in $W^{1,2}(\epsilon, R)$ where $\varepsilon \in(0, R)$. It is clear that for any $\epsilon \in(0, R),\left\{u_{m}\right\}$ is bounded in $W^{1,2}(\epsilon, R)$. We may get that $u_{m} \rightarrow u$ uniformly over $[\epsilon, R]$ as $m \rightarrow \infty$ applying the compact embedding $W^{1,2}(\epsilon, R) \rightarrow C[\epsilon, R]$. Thus, we have for any pair $r_{1}, r_{2} \in(0, R), r_{1}<r_{2}$, with (16), 


$$
\begin{aligned}
\left|u_{m}^{2}\left(r_{2}\right)-u_{m}^{2}\left(r_{1}\right)\right| & \leq 2\left(\int_{r_{1}}^{r_{2}} r u_{m, r}^{2}(r) \mathrm{d} r\right)^{1 / 2}\left(\int_{r_{1}}^{r_{2}} \frac{u_{m}^{2}(r)}{r} \mathrm{~d} r\right)^{1 / 2} \\
& \leq 2 C^{1 / 2}\left(\int_{r_{1}}^{r_{2}} \frac{u_{m}^{2}(r)}{r} \mathrm{~d} r\right)^{1 / 2}
\end{aligned}
$$

Taking $m \rightarrow \infty$, we get

$$
\left|u^{2}\left(r_{2}\right)-u^{2}\left(r_{1}\right)\right| \leq 2 C^{1 / 2}\left(\int_{r_{1}}^{r_{2}} \frac{u^{2}(r)}{r} \mathrm{~d} r\right)^{1 / 2}
$$

Since $\frac{u^{2}}{r} \in L(0, R)$, the right-hand side of (23) tends to zero as $r_{1}, r_{2} \rightarrow 0$. Hence,

$$
\zeta_{0}=\lim _{r \rightarrow 0} u^{2}(r)=0
$$

As a consequence, the boundary condition $u(0)=0$ is achieved. With (13), $u$ is a solution to (13), and there is a real number $\alpha$ such that $(u, \alpha)$ satisfies (12).

Moreover, we may suppose that there is a point $r_{0} \in(0, R)$ such that $u\left(r_{0}\right)=0$, then $u_{r}\left(r_{0}\right)=0$ since $r_{0}$ is a minimum point for the function $u(r)$. By the uniqueness theorem of the initial value problem of ordinary differential equations, we have $u(r)=0$ for all $r \in(0, R)$, thus contradicting the fact $\Phi(u)=\Phi_{0}>0$. Hence, $u(r)>0$ for all $r \in(0, R)$.

2) We establish

$$
\liminf _{r \rightarrow 0}\left\{r u(r)\left|u_{r}(r)\right|\right\}=0
$$

Suppose otherwise that (24) is not valid, equivalently, $\liminf _{r \rightarrow 0}\left\{r u(r)\left|u_{r}(r)\right|\right\} \neq 0$, then there is a $\epsilon_{0}>0$ and $r_{0} \in(0, R]$ so that $r u(r)\left|u_{r}(r)\right| \geq \epsilon_{0}$ for all $r \in\left(0, r_{0}\right)$. However,

$$
\infty=\int_{0}^{r_{0}} \frac{\epsilon_{0}}{r} \mathrm{~d} r \leq \int_{0}^{r_{0}} u\left|u_{r}\right| \mathrm{d} r \leq\left(\int_{0}^{r_{0}} \frac{u^{2}}{r} \mathrm{~d} r\right)^{1 / 2}\left(\int_{0}^{r_{0}} r u_{r}^{2} \mathrm{~d} r\right)^{1 / 2}
$$

which contradicts with $\varepsilon(u)<\infty$. So, (24) is valid. From (24), we can find a sequence $\left\{r_{j}\right\}$ such that $r_{j} \rightarrow 0$ as $j \rightarrow \infty$ and

$$
\lim _{j \rightarrow \infty}\left\{r_{j} u\left(r_{j}\right) u_{r}\left(r_{j}\right)\right\}=0
$$

Multiplying (5) by $u$, integrating over $\left[r_{j}, R\right]$, letting $j \rightarrow \infty$. Appealing to (26), we obtain

$$
-\int_{0}^{R} r u_{r}^{2} \mathrm{~d} r=\int_{0}^{R}\left\{\frac{n^{2}}{r} u^{2}+2 \alpha r u^{2}-2 r u^{2}+\frac{2 r u^{2}}{\sqrt{1+u^{2}}}\right\} \mathrm{d} r
$$

Using $\left(\sqrt{1+u^{2}}-1\right)^{2} \leq \frac{1}{4} u^{4}$, we have that

$$
\begin{aligned}
-\int_{0}^{R} r u_{r}^{2} \mathrm{~d} r & =\int_{0}^{R}\left\{\frac{n^{2}}{r} u^{2}+2 \alpha r u^{2}-2 r u^{2}+\frac{2 r u^{2}}{\sqrt{1+u^{2}}}\right\} \mathrm{d} r \\
& \geq \int_{0}^{R}\left\{\frac{n^{2}}{r} u^{2}+2 \alpha r u^{2}-2 r u^{4}\right\} \mathrm{d} r \geq \int_{0}^{R}\left(\frac{n^{2}}{r^{2}}+2 \alpha\right) r u^{2} \mathrm{~d} r-2 \int_{0}^{R} r u^{4} \mathrm{~d} r
\end{aligned}
$$


We may treat $u$ as a radially symmetric function defined over $\mathbb{R}^{2}$ with its support contained in the disk $B_{R}$. Hence, from the classical $G-N$ inequality over $\mathbb{R}^{2}$, we deduce

$$
\int_{0}^{R} r u^{4} \mathrm{~d} r \leq 4 \pi \int_{0}^{R} r u^{2} \mathrm{~d} r \int_{0}^{R} r u_{r}^{2} \mathrm{~d} r
$$

with $\Phi(u)=\Phi_{0}$, we have

$$
\left(4 \Phi_{0}-1\right) \int_{0}^{R} r u_{r}^{2} \mathrm{~d} r-\int_{0}^{R}\left(\frac{n^{2}}{r^{2}}+2 \alpha\right) r u^{2} \mathrm{~d} r \geq 0
$$

Therefore, when $\Phi_{0} \leq \frac{1}{4}, \frac{n^{2}}{r^{2}}+2 \alpha>0$ for $r \in(0, R], u \equiv 0$. as claimed.

\section{Conclusion}

Through the prove of the theorem 2.2, we get that the existence of positive solution pairs $(u, \alpha)$ by a constrained minimization approach. In other words, we get the existence of ring-profiled optical vortex solitons propagating in a square-root nonlinear media. Moreover, we obtain that there is no nontrivial small-energy-flux solution satisfying $\Phi(u)=\Phi_{0} \leq 1 / 4$, if $n^{2}+2 r^{2} \alpha>0$ for $r \in[0, R]$.

\section{Conflicts of Interest}

The author declares no conflicts of interest regarding the publication of this paper.

\section{References}

[1] Adhikari, S.K. (2010) Localization of a Bose-Einstein Condensate Vortex in a Bichromatic Optical Lattice. Physical Review A, 81, Article ID: 043636.

https://doi.org/10.1103/PhysRevA.81.043636

[2] Davydova, T.A. and Yakimenko, A.I. (2004) Stable Multi-Charged Localized Optical Vortices in Cubicquintic Nonlinear Media. Journal of Optics A: Pure and Applied Optics, 97, S197-S201. https://doi.org/10.1088/1464-4258/6/5/010

[3] Kartashov, Y.V., Vysloukh, V.A. and Torner, L. (2004) Rotary Solitons in Bessel Optical Lattices. Physical Review Letters, 93, Article ID: 093904. https://doi.org/10.1103/PhysRevLett.93.093904

[4] Kartashov, Y.V., Vysloukh, V.A. and Torner, L. (2005) Stable Ring Vortex Soltions in Bessel Optical Lattices. Physical Review Letters, 94, Article ID: 043902. https://doi.org/10.1103/PhysRevLett.94.043902

[5] Mamaev, A.V., Saffman, M. and Zozulya, A.A. (1996) Propagation of Stripe Beams in Nonlinear Media: Snake Instability and Creation of Optical Vortices. Physical Review Letters, 76, 2262-2265. https://doi.org/10.1103/PhysRevLett.76.2262

[6] Neshev, D., Alexander, T.J., Ostrovskaya, E.A., Kivshar, Y.S., Martin, H., Makasyuk, I. and Chen, Z. (2004) Observation of Discrete Vortex Solitons in Optically-Induced Photonic Lattices. Physical Review Letters, 92, Article ID: 123903. https://doi.org/10.1103/PhysRevLett.92.123903

[7] Rozas, D., Law, C.T. and Swartzlander Jr., G.A. (1997) Propagation Dynamics of Optical Vortices. Journal of the Optical Society of America B, 14, 3054-3065. 
https://doi.org/10.1364/JOSAB.14.003054

[8] Skryabin, D.V. and Firth, W.J. (1998) Dynamics of Self-Trapped Beams with Phase Dislocation in Saturable Kerr and Quadratic Nonlinear Media. Physical Review E, 58, 3916-3930. https://doi.org/10.1103/PhysRevE.58.3916

[9] Lin, T.C., Belić, M.R., Petrović, M.S., Hajaiej, H. and Chen, G. (2017) The Virial Theorem and Ground State Energy Estimates of Nonlinear Schrödinger Equations in $\mathbb{R}^{2}$ with Square Root and Saturable Nonlinearities in Nonlinear Optics. Calculus of Variations and Partial Differential Equations, 56, Article No. 147. https://doi.org/10.1007/s00526-017-1251-4

[10] Liu, C. and Ren, Q. (2015) On the Steady-State Solutions of a Nonlinear Photonic Lattice Model. Journal of Mathematical Physics, 53, Article ID: 031501. https://doi.org/10.1063/1.4914333

[11] Petroskia, M.M., Petrovica, M.S. and Belica, M.R. (2007) Quasi-Stable Propagation of Vortices and Soliton Clusters in Previous Saturable Kerr Media with Square-Root Nonlinearity. Optics Communications, 279, Article ID: 196202. https://doi.org/10.1016/j.optcom.2007.07.006

[12] Skarka, V., Berezhiani, V.I. and Boucher, V. (2000) Self-Trapping of Electromagnetic Pulses in Narrow-Gap Semicon-Ductors. Physical Review B, 61, Article ID: 10201. https://doi.org/10.1103/PhysRevB.61.10201

[13] Yang, Y. and Zhang, R. (2014) Existence of Optical Vortices. SIAM Journal on Mathematical Analysis, 46, 484-498. https://doi.org/10.1137/120894105 